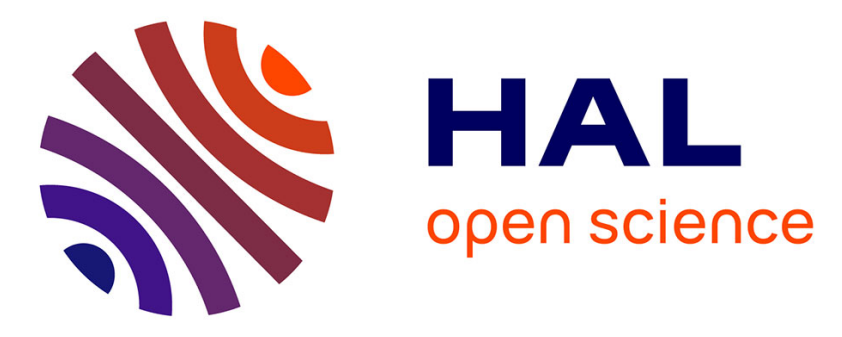

\title{
A New Objective Function For Hybrid Network In The Smart Grid
}

\author{
François Lemercier, Nicolas Montavont, Laurent Toutain, Philippe
}

Chiummiento

\section{- To cite this version:}

François Lemercier, Nicolas Montavont, Laurent Toutain, Philippe Chiummiento. A New Objective Function For Hybrid Network In The Smart Grid. WoWMoM 2018: IEEE 19th International Symposium On a World Of Wireless, Mobile and Multimedia Networks, Jun 2018, Chania, Greece. 10.1109/WoWMoM.2018.8449744 . hal-01923902

\section{HAL Id: hal-01923902 \\ https://hal.science/hal-01923902}

Submitted on 15 Nov 2018

HAL is a multi-disciplinary open access archive for the deposit and dissemination of scientific research documents, whether they are published or not. The documents may come from teaching and research institutions in France or abroad, or from public or private research centers.
L'archive ouverte pluridisciplinaire HAL, est destinée au dépôt et à la diffusion de documents scientifiques de niveau recherche, publiés ou non, émanant des établissements d'enseignement et de recherche français ou étrangers, des laboratoires publics ou privés. 


\title{
A New Objective Function For Hybrid Network In The Smart Grid
}

\author{
F. Lemercier \\ Itron, IMT Atlantique, IRISA, \\ 2 rue de la Châtaigneraie \\ 35576 Cesson Sevigné, France \\ francois.lemercier@imt-atlantique.fr
}

\begin{abstract}
Using multiple heterogeneous network interfaces in a smart grid may help in providing a better quality of service and increase the reliability of the communication framework. We propose to add a wireless interface to the existing PLC interface and rely on RPL, a well-known routing protocol for the Internet of Things, that has been designed to support the constraints of low power and lossy networks. We propose a new algorithm to manage multiple interfaces and study the impact of combining a radio and a PLC interface in a smart metering architecture. Using discrete event simulation, we show how the network can be more resilient to failure while the overall quality of service can be significantly increased.
\end{abstract}

Index Terms-RPL; LLN; Hybrid Routing; Smart Grid; Heterogeneous networks

\section{INTRODUCTION}

A significant part of the evolution in the electricity grid relies on an overlay information communication network that is inspired from the Internet and the Internet of Things (IoT). New services require higher bandwidth, high reliability and secure information exchange. The Power Line Communication (PLC) network used in the Automatic Meter Reading (AMR) enables automatic and remote electric and gas meter readings. The new generation of the electric grid, called the smart grid, is an electric power distribution network based on Advanced Metering Infrastructure (AMI) that provides a two-way communication between providers and customers.

PLC communication networks are however subject to high sensitivity to interference. Severe noise condition is common and require a MAC protocol that can deal with high variation of available bandwidth. Despite this dynamic adaptation, alternative communication technologies are considered, such as Wireless Sensor Network, Mesh, cellular, Wimax, IoT Long Range, etc. Most of them are considered Low Power and Lossy networks (LLN) because they are highly sensitive to noise and may not work in specific environments. In order to cope with all client scenarios, and to provide universal network access at a certain level of quality of service, most of future smart meter will embed several heterogeneous communication technologies. Thus there is a challenge in supporting these multiple interfaces.

Note that most of these technologies are short range, and require multiple hops to reach a given destination. Routing protocols play a major role by optimizing the selected paths according to application requirements and field specificity. RPL[1] is the most popular routing protocol in the IoT community. Contrary to AODV [2] or LOAD [3], RPL is a proactive protocol based on distance vector. RPL builds a tree rooted at a sink by leaving each node to choose its next hop toward the root. This selection is based on an Objective Function (OF) that determines the best relay node and computes the node rank that represents more or less the distance from the root.

RPL has been designed to operate on single communication network and is mostly used on homogeneous network. In [4] we present three different solutions to modify RPL to benefit from an hybrid network. One of the given solution, called Parent Oriented (PO), merges the characteristics of the heterogeneous interfaces to provide a single virtual link with a neighbor in order to compare potential parent during the RPL selection operation. In this paper, we show how the Parent Oriented solution could increase the network performance by handling an additional RF interface to a smart meter, under a PLC smart grid application scenario.

\section{RELATED WORK}

RPL [1] is a routing protocol that organizes the topology in a Directed Acyclic Graph (DAG). Vertices between nodes have a direction and a "non-circular" property. The routing graph has at least one root, which is a node with no outgoing edge. RPL uses the destination oriented DAG (DODAG) to construct topologies, where only one root is defined.

Each node in the DAG is assigned a rank to indicate a virtual position. Except the root, all nodes start with an infinite rank. At the initialization phase, the root sends a multicast DIO message to its neighbors. Nodes in range will receive and process this DIO message. Consequently, they will choose the root as its DODAG parent and compute their rank with a given OF. Then these receiving nodes will send themselves a multicast DIO message including their new rank. DIO messages that include a higher rank than the receiving node rank, are ignored. While there is a good litterature on RPL and its performance over a (single interface) wireless network, only few works have studied RPL over a PLC network, which has its own specific characteristics [5]. Moreover, only few works have been done using both PLC and wireless communications in a RPL network. 
Ben-Shimol et al. [6] present an evaluation of RPL in large scale PLC networks using field measurement to configure the simulation channel. They proposed several improvements of RPL based on OF modifications and rank computation to address the constrains of the PLC network. Their contribution shows improvements in the DODAG formation, but at the cost of more control messages for DODAG maintenance, which raises the issue of large scale PLC networks. Ben Saad et al. [7] propose an heterogeneous architecture based on RFPLC gateways to make a cooperation between RF-only and PLC-only sensors. Such an architecture confers improvements in network lifetime and reliability in small topology but could lead to severe congestion around gateways in large scale scenario.

\section{MANAGING Heterogeneous INTERFACES IN RPL}

In this paper, we consider that every node in the network is potentially able to communicate via two heterogeneous interfaces, each having their own link qualities with their neighbors. For each neighbor, we store the monitored link quality for each interface in a neighbor table. From these two link qualities, we compute a new metric that allows to determine the best neighbors.

\section{A. The Parent Oriented (PO) solution}

We propose to modify the way RPL selects a parent by considering a node ability to communicate on multiple interfaces. During the parent selection phase, potential parents that have only one interface will be downgraded comparing to multiinterfaces candidates. To do so, we compute the hybrid metric when the OF is called to calculate the rank of the node. If the neighbor is accessible only with one technology, the missing metric will be set to a default value, similar to a broken link.

To increase the stability of the DODAG, when a preferred parent node looses one of its interface because of a failure, we propose to keep it as the preferred parent until the OF is called again for a parent selection.

Once the hybrid parent is chosen, it has the possibility to forward message on either of the two available interfaces. If a node can communicate with a potential parent through a hybrid link, the node will select the interface with the best metric for the forwarding.

\section{B. Retransmission}

Both PLC and radio interfaces can support multiple modulations. The modulation is chosen by the MAC layer depending on the link quality. The better the link quality, the faster the chosen modulation. When a parent node is registered in a routing table, all possible modulations are stored for each interface (if available). To address a transmission failure on hybrid nodes, we propose the following algorithm. Let us consider interface $\mathrm{A}$ as the best interface and interface $\mathrm{B}$ the other one. If a node sends a packet with interface $\mathrm{A}$, which has the best metric of the two technologies available, and this attempt fails, the first re-transmission happens with the interface A but with the lowest modulation registered in the

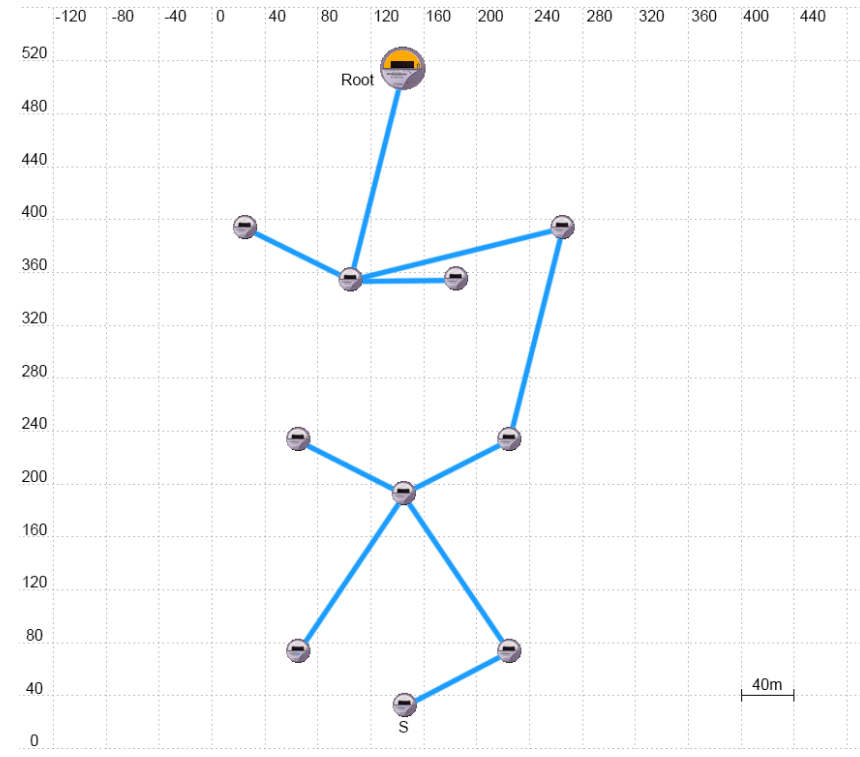

Fig. 1. Hybrid Topology example

routing table. If this second attempt fails again, the node will change to interface $\mathrm{B}$ and select the best modulation registered for this interface. In case of another fail, the node will select the lowest transmission available on interface B.

\section{Simulation PLATFORM}

To evaluate the performance of the Parent Oriented solution, we have created a smart grid scenario that consists of a simple data collecting application. We have developed a new framework in the Riverbed Modeler 18.5 network simulator. After the initialization phase where all nodes define their preferred parent toward the root, node S (see Fig.1) periodically sends data packets to the root. We compared two different traffic scenarios going from one meter reading every hours to smart meter reading every minutes. Our evaluation model considers hybrid nodes with a network layer, where RPL operates, that shares two separate MAC / Physical layers. Thus, the node could send and receive message via PLC and Wireless $802.15 .4 \mathrm{e} / \mathrm{g}$. We use a smart controller between the MAC and the network layer that is in charge of the radio and PLC link evaluation and the retransmission policy scheme.

Each scenario consists of a topology made of eleven routers. The PLC has an attenuation of $9 \mathrm{~dB} / \mathrm{m}$ and $3 \mathrm{db}$ at derivations. Simulation parameters are summarized in table I. We also implemented the Interface Oriented (IO) algorithm presented in [4] that selects the parent with the interface that has the best metric. This solution considers each link as a potential parent, and a neighbor with only a single interface is not penalized. After a given time, we consider the DAG stable enough and we analyze how nodes react to the failure of a given node PLC interface. Note that RPL is a dynamic protocol and constantly adapts to the link quality, link failure and link creation.

Firstly, figure 2 shows the average of the number of parent changes that occur in the whole DODAG. As the Parent Oriented (PO) solution keeps a parent even if it loses an 


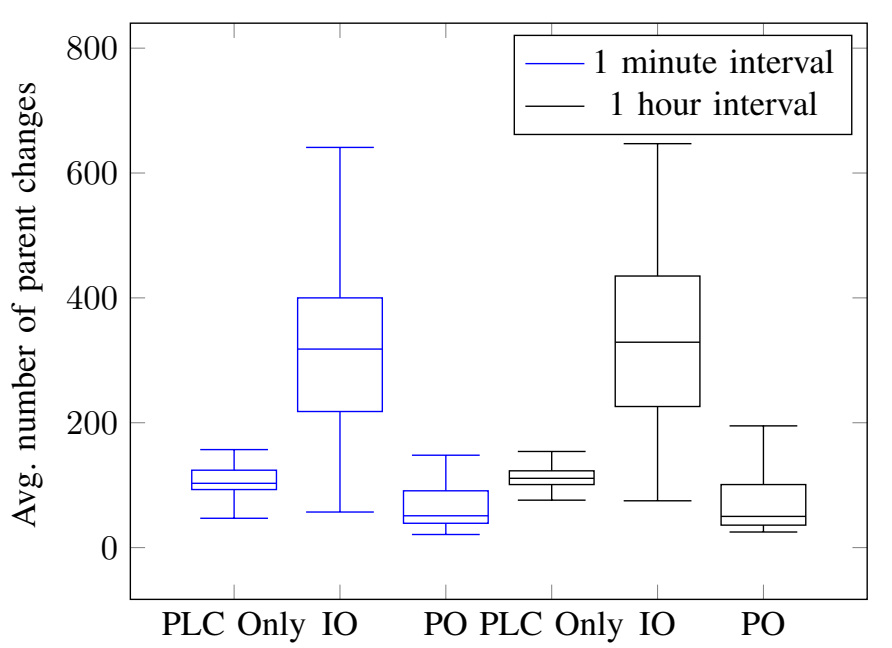

Fig. 2. Average number of parent changes

\begin{tabular}{|c|c|}
\hline RPL Obbjective function & Parent Oriented / Interface Oriented \\
\hline Radio environment & $802.15 .4 \mathrm{e} / \mathrm{g}$ \\
\hline PLC environment & P1901.2, one phase \\
\hline Number of routers & 11 \\
\hline Distance between routers & 80 to $120 \mathrm{~m}$ \\
\hline Runs & 100 \\
\hline Packet interval time & 1 hour $/ 1 \mathrm{~min}$ \\
\hline
\end{tabular}

SIMULATION PARAMETERS

interface, it offers the best stability comparing to the other schemes. The Interface Oriented solution, selecting the best metric without considering the hybridity of the node results in an unstable DODAG. However, as the parent selection is also governed by a rank stretching to lower the parent switching phenomenon, carefully selecting the rank stretching value could increase the stability of the DODAG at the cost of keeping a parent with the non optimal metric. Secondly, we analyze the number of sent DIO messages to maintain the DODAG. We show in Fig. 3 that hybrid networks considerably reduce the number of RPL messages in this scenario, taking benefit from the stability of the radio network. The End-toend delay has been also evaluated with different application scenarios. As our $\mathrm{OF}$ has been designed to optimize the stability, we observe that the average number of hops is slightly greater than a single interface network. This results in a minor increase in the delay.

\section{CONCLUSION AND FUTURE WORK}

In this paper, we presented a solution to manage hybrid network in RPL for smart grids. Using the Parent Oriented solution, we compare potential parents and encourage the selection of hybrid nodes as preferred parent. For nodes which have a single interface, we increase the merged link quality with that neighbor in order to decrease its attractiveness.

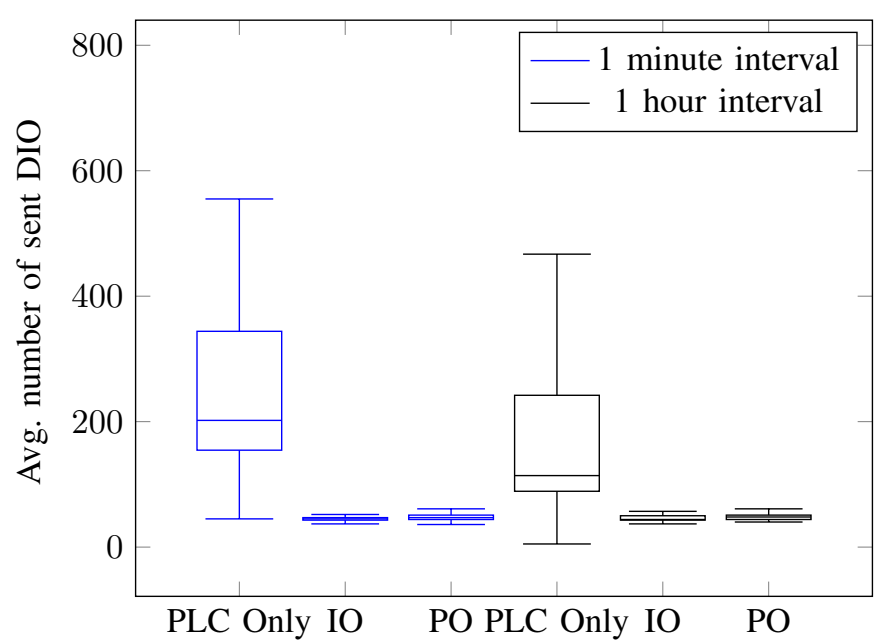

Fig. 3. Average number of sent DIO message

We also presented a re-transmission scheme that takes into account the number of interfaces of a node, to help a node not to change its preferred parent too frequently.

By means of simulations using realistic PLC and RF channels, we showed that PLC networks can be significantly optimized using hybrid nodes.

A future direction is to implement a multiple DODAG / Instance algorithm to have a specific Objective Function for each network specificity. Another direction would be also to fully use the potential of the hybrid network by sending duplicate data on both interfaces.

\section{ACKNOWLEDGMENT}

The author would like to thank Professor N. Montavont and Doctor L. Toutain from IMT Atlantique, IRISA, for their supervision and support and P. Chiummiento, CTO, from Itron France for his precious help.

\section{REFERENCES}

[1] T. Winter, P. Thubert, A. Brandt, J. Hui, R. Kelsey, P. Levis, K. Pister, R. Struik, JP. Vasseur, and R. Alexander. RPL: IPv6 Routing Protocol for Low-Power and Lossy Networks. RFC 6550 (Proposed Standard), March 2012.

[2] Charles E. Perkins, Elizabeth M. Belding-Royer, and Samir R. Das. Ad hoc on-demand distance vector (AODV) routing. RFC, 3561:1-37, 2003.

[3] Soohong Daniel Park. 6LoWPAN Ad Hoc On-Demand Distance Vector Routing (LOAD). (draft-daniel-6lowpan-load-adhoc-routing-03), June 2007. Work in Progress.

[4] Francois Lemercier, Nicolas Montavont, Laurent Toutain, Kumaran Vijayasankar, Ramanuja Vedantham, and Philippe Chiummiento Itron. Support for hybrid network in RPL. pages 527-532, 2016.

[5] Yocheol Kim, Jung Nam Bae, and Jin Young Kim. Performance of power line communication systems with noise reduction scheme for smart grid applications. IEEE Trans. Consumer Electronics, 57(1):46-52, 2011.

[6] Y. Ben-Shimol and Y. Zohar. Routing in large realistic plc smart-grids. pages 1-6, April 2017.

[7] Leila Ben Saad, Cedric Chauvenet, and Bernard Tourancheau. Heterogeneous IPv6 Infrastructure for Smart Energy Efficient Building. September 2011 . 